\title{
Effective ureteral access sheath insertion during flexible ueteroscopy: Influence of the ureteral orifice configuration
}

\author{
Raed A. Azhar ${ }^{1,2}$; Musab M. Alghamdi' ${ }^{2}$ Abdullah A. Khawaji ${ }^{2}$; Anmar M. Nassir ${ }^{3}$; \\ Sameer Munshi ${ }^{4}$; Waseem Tayeb ${ }^{4}$; Mohamed A. Elkoushy ${ }^{4,5}$ \\ ${ }^{1}$ Department of Urology, King Abdul-Aziz University, Jeddah, Saudi Arabia; ${ }^{2}$ Department of Urology, \\ International Medical Center, Jeddah, Saudi Arabia; ${ }^{3}$ Department of Surgery, Umm-Alqura University, \\ Mecca, Saudi Arabia; ${ }^{4}$ Department of Surgery, King Abdullah Medical City, Mecca, Saudi Arabia; \\ ${ }^{5}$ Department of Urology, Suez Canal University, Ismailia, Egypt
}

Cite as: Azhar RA, Alghamdi MM, Khawaji AA, et al. Effective ureteral access sheath insertion during flexible ueteroscopy: Influence of the ureteral orifice configuration. Can Urol Assoc J 2022 February 28; Epub ahead of print. http://dx.doi.org/10.5489/cuaj.7656

Published online February 28, 2022

Corresponding author: Dr. Raed A. Azhar, Department of Urology, Faculty of Medicine, King Abdul-Aziz University, Jeddah, Saudi Arabia; raedazhar@gmail.com

$* * *$

\section{Abstract}

Introduction: We sought to determine the possible predictors for effective insertion of the ureteral access sheath (UAS) during flexible ureteroscopy (fURS) in virgin ureters and its impact on postoperative ureteral wall injury and the procedural outcome.

Methods: A retrospective review of prospectively collected data was performed for all consecutive patients scheduled for fURS of virgin ureters at two tertiary care centers between 2018 and 2020. Demographics, stone characteristics, and perioperative data, including the configuration of the ureteral orifice (UO) over introductory guidewire insertion, were collected. Multivariate logistic regression was used to detect possible predictors of successful UAS insertion.

Results: In total, 128 patients who underwent primary fURS were included, with a mean age of $43.3 \pm 12.3$ years and a stone burden of $12.3 \pm 6.9 \mathrm{~mm}$. One hundred and seven patients $(85.9 \%)$ achieved successful ureteral access insertion, including $81(63.3 \%)$ without ureteral dilatation and $29(22.7 \%)$ out of the $35(27.3 \%)$ patients who needed ureteral dilation. Patients who underwent successful UAS placement into virgin ureters were significantly older and had a lower body mass index. A tent-shaped UO over the guidewire led to successful UAS insertion. In multivariate regression analysis, cases with 
body mass index (BMI) $<30 \mathrm{~kg} / \mathrm{m}^{2}$ (odds ratio [OR] 1.89, 95\% confidence interval [CI] 1.28-7.03) and those with a tent-shaped UO over the introductory guidewire (OR 6.60, 95\% CI 3.8-7.2) maintained their significance to predict successful UAS insertion into virgin ureters. Nine patients $(8.2 \%)$ had ureteral mucosal injuries, and the overall stonefree rate was $78.2 \%$.

Conclusions: Patients with normal BMIs and tent-shaped UOs over the introductory guidewires are more likely to achieve primary UAS insertion without the need for ureteral dilation.

\section{Introduction}

Flexible ureteroscopy (fURS) has dramatically changed and improved the minimally invasive management of complex nephrolithiasis. This technology was further supported by the introduction of the ureteral access sheath (UAS), which improved the costeffectiveness of the procedure. The UAS improves operative vision, enables repeated passage of the ureteroscope while minimizing damage to the ureter, improves the irrigation fluid, facilitates extraction of small stone fragments, and decreases intrarenal pressure. ${ }^{1-4}$

However, this technique remains challenging for some urologists, ${ }^{5}$ and its usefulness during ureteroscopic stone removal is still debatable. The results from a metaanalysis did not support the use of a UAS during fURS due to increased postoperative complications with comparable stone-free rates (SFRs), operative times, and intraoperative adverse events. ${ }^{6}$ Furthermore, placement of the UAS carries an increased risk of ureteral wall ischemia and injury with consequent ureteral strictures. Nevertheless, preoperative ureteral stenting seems to increase the success rate of UAS placement during retrograde intrarenal surgery (RIRS), with a tendency to use a larger sheath. ${ }^{7}$

Some authors have reported improved SFR with UAS placement before fURS ${ }^{\mathbf{8}}$, while others support its systematic use ${ }^{\mathbf{3}, \mathbf{9}}$ with an $8.6-22 \%$ failure rate of insertion. ${ }^{\mathbf{1 0 1 1}}$ Effective insertion of UAS is significantly associated with preoperative ureteral stenting and a history of previous ipsilateral URS. ${ }^{10,11}$ However, no factors have been identified to predict UAS insertion in virgin ureters. ${ }^{12}$

We believe that other perioperative variables may influence effective UAS insertion, including the surgeon's experience and preoperative medication with $\alpha$ adrenergic blockers, which would relax and relatively dilate the intramural distal ureter. In addition, some experts have reported the impact of the ureteral orifice (UO) shape over a stiff guidewire on the success of UAS introduction, which has not been investigated. If the UO stays round and narrow around the stiff wire, there is no need to try UAS 
placement, but if the UO opens like a tent, this is a good indicator for UAS placement. Furthermore, determining whether UAS insertion will be successful, in case the surgeon would like to use, might also have financial implications.

The aim of this study was to assess the predictors for effective UAS insertion and to assess its impact on the outcome of the procedure, which may improve patient counseling and guide surgeons in deciding whether to proceed, apply pre-stenting, or abort the procedure.

\section{Methods}

\section{Study design}

A retrospective review of prospectively collected data was performed for all consecutive patients undergoing primary fURS for the management of renal stones at two tertiary care centers between 2018 and 2020. Experienced and fellowship-trained endourologists carried out all interventions.

Patients over 18-years old who were considered good surgical candidates and were able to provide written informed consent were included. Patients were excluded if they had ipsilateral ureteral stones or strictures, previous ipsilateral URS or ureteral stents, history of bilharziasis or tuberculosis, or complete ipsilateral ureteral duplication. Patients with active urinary tract infections were also temporarily excluded until they had proven negative urine cultures.

All patients underwent a preoperative, complete laboratory workup to assess comorbid conditions and fitness for anesthesia, including complete urinalysis and culture, complete blood count, kidney and liver function tests, random blood sugar analysis, bleeding profiles, and electrocardiography. Preoperative nonenhanced spiral computed tomography (NECT) was also performed on all patients.

\section{Data collection}

Demographic data, including age, sex, body mass index (BMI), concomitant preoperative use of $\alpha$-adrenergic blockers, history of previous ipsilateral URS or ureteral stenting, ASA score, stone size (the largest stone diameter calculated on NECT), number of stones, and stone location (coded as pelvis, inferior calyx, middle calyx and upper calyx), were collected. Perioperative parameters included operative time, use of a UAS, need for ureteral dilatation, shape of the ureteral orifice (UO) over an introductory guidewire (Figure 1), intracorporeal laser lithotripsy (ICLT), postoperative stenting, and perioperative complications.

\section{Operative procedure}

All patients received preoperative prophylactic parenteral antibiotics, and all procedures were performed under general anesthesia. Cystoscopy was started with identification of 
the shape of both UOs pre- and post- introduction of guidewires. Retrograde ureteropyelography was performed to check the status of the ureter, with the introduction of a safe hydrophilic guidewire in the upper tract in all cases. Alongside a second safety guidewire, a UAS (Navigator 11/13Fr, Boston Scientific, Natik, MA, USA) was passed up to the proximal ureter under fluoroscopy guidance. If UAS placement was impossible, atrial ureteral dilatation was performed, and if this in turn was not possible, a sheathless procedure was attempted. If this last attempt failed, a pigtail, double-J ureteral catheter was left in place for passive dilatation, and the procedure was postponed. After successful insertion of the UAS, URS was then conducted with ICLT, when indicated. A postoperative ureteral stent was placed at the end of the procedure. Systematic visual assessment of the entire ureter was performed with digital fURS at the end of the procedure or during removal of the UAS to recognize and grade ureteral injury.

\section{Primary and secondary endpoints}

Patients were stratified according to the results into three groups: effective passage of the UAS without a need for UO dilation, effective passage of the UAS after sequential UO dilation, and failure to pass the UAS with or without dilatation. The primary endpoint was to determine the possible predictors for effective insertion of a UAS into virgin ureters. The secondary endpoints were to assess UAS-related postoperative ureteral wall injury, the SFR, and associated ureteral injuries, which were evaluated according to Traxer and Thomas, 2013.13

\section{Followup data}

Patients were evaluated one and 3 months postoperatively with NECT to evaluate the stone-free status, defined as complete absence of stone fragments or the presence of a single residual non-obstructing fragment less than $4 \mathrm{~mm}$. Hospital stays after the procedures were not considered serious adverse events unless the hospital admissions occurred because of complications from the performed procedure.

\section{Data analysis}

Data were collected and tabulated using the commercially available Statistical Package for the Social Sciences software (SPSS Inc., Chicago, IL, USA), version 22. Descriptive statistics were presented in terms of frequency, percentage, means and standard deviations. Differences between groups were compared with Fisher's exact test for categorical variables and Student's $t$-test for continuous variables. The interplay of more than two variables was analyzed using multivariate logistic regression to detect possible predictors of successful UAS insertion. Multivariate model included all the clinically important variables, which would potentially influencing the outcome of interest, 
irrespective of their statistical significance in the univariate model. Two-tailed p-values of less than 0.05 were set for statistical significance.

\section{Results}

A total of 128 patients who underwent primary flexible URS were included, with a mean age of $43.3 \pm 12.3$ years and a stone burden of $12.3 \pm 6.9 \mathrm{~mm}$. Ninety-five $(74.2 \%)$ patients were males. The shape of the UO over the introductory guidewire was documented in 92 patients $(71.9 \%)$, including $56(60.9 \%)$ round UOs and $36(39.1 \%)$ tent-shaped orifices. Baseline demographic and stone characteristics of the study population are presented in Table 1.

One hundred and seven patients $(85.9 \%)$ had successful ureteral access insertion, including $81(63.3 \%)$ without ureteral dilatation and $29(22.7 \%)$ out of the $35(27.3 \%)$ patients who needed ureteral dilation. The procedure was aborted in 12 patients $(9.4 \%)$ after insertion of double-J ureteral stents.

Patients who underwent successful UAS placement in virgin ureters were significantly older, had a lower BMI, and had significantly right-sided stones (Table 2). Compared to round UOs, tent-shaped orifices over the guidewires facilitated successful insertion of UAS, even without ureteral dilatation (94.4 vs. 71.4, $\mathrm{p}=0.007)$. Patient sex, previous history of stone passage, stone burden, stone multiplicity and concomitant use of $\alpha$-adrenergic blockers did not influence the successful insertion of the primary UAS (Table 2).

UAS successfully passed without dilatation in older patients with lower BMI who had a tent-shaped UO, compared to those who underwent ureteral dilatation (Table 3). Otherwise, successful insertion of the UAS was not influenced by other patient or stone characteristics. The overall SFR after the primary single URS was $78.2 \%$, which was comparable between patients with primary UAS insertion and those without primary UAS insertion $(79.0 \%$ vs. $75.8 \%, \mathrm{p}=0.79)$.

The multivariate model included patient age, gender, BMI, history of spontaneous passage of stones, preoperative use of $\alpha$-adrenergic blockers, and shape of UO over the introductory guide wire. In the multivariate regression analysis, cases with BMIs of <30 $\mathrm{kg} / \mathrm{m}^{2}$ [OR (95\% CI): $\left.1.89(1.28-7.03)\right]$ and those with a tent-shaped UO over the introductory guidewire [OR (95\% CI): 6.60 (3.8-7.2)] maintained their significance to predict successful UAS insertion in virgin ureters (Table 4). Out of 110 patients, intraoperative adverse events were recorded in 9 (8.2\%), among whom 7 (6.4\%) patients had ureteral mucosal erosion without smooth muscle injury (grade 1) and $2(1.8 \%)$ patients had mucosal and smooth muscle injuries with preserved adventitia (grade 2). No adventitial perforation or ureteral avulsion was detected (Table 5).

\section{Discussion}


Stone-free status is the primary goal in the management of patients with urolithiasis. The usefulness of a UAS during ureteroscopic stone removal is still debatable. Some authors have reported improved SFR with UAS placement before fURS,${ }^{8}$ while other studies support its systematic use, ${ }^{3,9}$ with an $8.6-22 \%$ failure rate of insertion. ${ }^{10,11}$ Evidence from a meta-analysis did not support the use of UAS during URS, as it did not improve the operative outcomes but was possibly associated with higher postoperative complications. ${ }^{6}$ However, only two randomized controlled studies were included in the latter analysis.

Nevertheless, a UAS may improve operative vision, improve irrigation fluid, decrease intrarenal pressure and enable repeated passage of the ureteroscope while minimizing damage to the ureter. ${ }^{1-4}$ These concepts encourage researchers to look for possible parameters that may enable effective insertion of UASs. The current study included patients with virgin ureters precluding preoperative ureteral stenting or a history of previous ipsilateral URS, which were significantly associated with successful UAS placement. ${ }^{10,11}$ The American Urological Association (AUA) and European Association of Urology (EAU) guidelines do not recommend routine preoperative stenting before URS. However, preoperative stenting may enhance successful UAS insertions and prevent associated ureteral wall injuries. ${ }^{11,13,14}$ Traxer and Thomas found that the absence of preoperative stenting was the most significant predictor for severe injuries associated with UAS placement (by sevenfold). ${ }^{13}$ Similarly, Breda and colleagues reported that prestented status was the only independent predictor for successful UAS insertion (98.5\% vs. $82 \%) .{ }^{15}$ Yuk and colleagues found that preoperative ureteral stenting seems to increase the success rate of UAS placement during RIRS, with a tendency to use a larger sheath. ${ }^{7}$ However, this does not affect operative time, perioperative complications or SFR.

Fuller and colleagues reported a 7.7\% failure rate for accessing the unstented ureter, even after ureteral dilatation. ${ }^{16}$ This failure rate is comparable to our figure after the exclusion of patients with aborted procedures. Unsuccessful ureteroscopic access in the former study was significantly lower in younger females (34 vs. 52 years). Compared to renal stones, proximal ureteral stones had a significantly higher failure rate for UAS $(18.3 \%)$ and remained the only significant predictor of access failure in the multivariable regression model compared with distal ureteral stones. ${ }^{16}$ It was not clear why the authors may have needed UAS insertion in cases with distal ureteral stones. Our results support successful UAS insertion in elderly patients, which did not maintain its significance in the multivariate model, as previously reported.

A recent study did not identify any patient demographics or stone characteristics that might influence the failure of UAS placement in virgin ureters. ${ }^{\mathbf{1 2}}$ The authors were unable to decide whether to proceed with URS, preoperative ureteral stenting, or abortion of the procedure. The latter study missed important factors that may influence UAS 
placement in a virgin ureter, such as the shape of the UO over the guidewire and concurrent use of $\alpha$-adrenergic antagonists. Moreover, the authors did consider the option of ureteral dilatation in the latter study. Preoperative $\alpha$-adrenergic blockers may inhibit peristalsis, with a subsequent reduction in ureteral pressure and maximal UAS insertion forces. ${ }^{17} \mathrm{Of}$ interest, non-stented patients who received preoperative $\alpha$-adrenergic antagonists for seven days had a significant reduction in UAS insertion forces and were comparable to pre-stented patients who did not receive these medications. ${ }^{17}$ Therefore, these medications seem to improve UAS-associated injuries, but it is still unknown whether UAS insertion-force reductions are dose dependent. This finding was not supported in our study, which may be because of the small number of patients who were on concomitant $\alpha$-adrenergic antagonists and failed UAS insertion, precluding any statistically significant differences.

In a multi-institutional prospective study, 1,494 (67\%) patients were treated with the use of a UAS, and $745(33 \%)$ were not. Although the difference was statistically comparable, SFRs were lower in the UAS group (73.9 vs. $82.8 \%$ ). Therefore, the UAS should not be primarily placed to increase the SFR. ${ }^{18}$ However, these results may be biased because UAS placement depends solely on the discretion of endourologists. Similarly, Berquet et al. ${ }^{9}$ and Kourambas et al. ${ }^{3}$ showed no significant differences in SFRs (86\% UAS vs. no UAS $87 \%$ ) and (79\% UAS vs. $86 \%$ no UAS), respectively.

Our results indicated that patients with normal BMIs seem to have a better chance for primary UAS insertion with or without UO dilatation. Hypothetically, URS can pose a challenge in obese patients due to difficulties in positioning and restriction of the surgeon's dexterity within the collecting system. However, Chew et al. concluded that the procedures are equally efficacious for obese and nonobese patients and that UAS placement in obese patients does not affect SFRs. ${ }^{19}$ Of interest, the preoperative tentshaped UO over a guidewire significantly predicts the successful insertion of UASs into virgin ureters by six to seven times, as detected in the multivariate model. To our knowledge, this variable was not assessed in previous similar studies and confirms our observations during surgical interventions.

Experimental studies showed that UAS placement would compress the ureter, resulting in decreased blood, ureteral ischemia and necrosis with subsequent ureteral thickening and stricture. ${ }^{\mathbf{2 0}, 21}$ The UAS may also increase the outer diameter of the ureter by approximately 2 -fold, resulting in severe overstretching of the ureteral tissue ${ }^{22}$ with a significant increase in the expression of the pro-inflammatory markers TNFa and COX$2 .{ }^{23}$ In 2013, Traxer and Thomas presented a reliable classification system to address intraoperative complications resulting from UAS placement. Following removal of UASs from 359 patients, the authors prospectively found superficial mucosal ureteral wall lesions in almost half of the patients following the insertion of a 12/14 Fr UAS, including 
$15 \%$ extending into the smooth muscle layer. ${ }^{13}$ They did not detect complete ureteral avulsions. In our study, no adventitial ureteral perforations or ureteral avulsions were detected in any of the 110 patients who underwent successful UAS insertion. Nine patients $(8.2 \%)$ had ureteral adverse events, most of them $(6.4 \%)$ had ureteral mucosal erosions without smooth muscle injury (grade 1), and only two patients had mucosal and smooth muscle injuries with a preserved adventitia (grade 2). It seems that tissue dynamics, such as resistance and elasticity, are influenced by potential injury rather than the physical narrowness of the ureter. ${ }^{11,13}$

This study is limited by its retrospective nature, and possible selection bias depended solely on the discretion of endourologists. The limited subgroup analyses may preclude possible significant differences in some variables, which may be assumed to increase successful UAS insertion, such as history of spontaneous stone passage and concurrent use of $\alpha$-adrenergic blockers. However, all the procedures were performed in tertiary care centers by many endourologists with different experience levels; hence, the results can be generalizable. To our knowledge, this is the first study to assess the influence of the shape of the UO over the introductory guidewire on successful UAS insertion. Notably, different commercially available UASs have different mechanical properties, which may influence the outcomes of their use. ${ }^{24}$

\section{Conclusions}

UAS insertion into virgin ureters seems to be influenced by BMI and the shape of the UO over the introductory wire. Patients with a normal BMI and a tent-shaped UO over the guidewire are more likely to achieve primary UAS insertion without the need for ureteral dilation. Surgeons should consider ureteral dilatation (rather than abortion of the procedure) and insertion of ureteral stents in patients with virgin ureteral orifices that seem to be inaccessible. 


\section{Predictors for effective insertion of the UAS during flexible URS}

\section{References}

1. Stern JM, Yiee J, Park S. Safety and efficacy of ureteral access sheaths. J Endourol 2007; 21:119-123.

2. Rehman J, Monga M, Landman J, et al. Characterization of intrapelvic pressure during ureteropyeloscopy with ureteral access sheaths. Urology 2003; 61:713718.

3. Kourambas J, Byrne RR, Preminger GM. Does a ureteral access sheath facilitate ureteroscopy? J Urol 2001; 165:789-793.

4. Auge BK, Pietrow PK, Lallas CD, et al. Ureteral access sheath provides protection against elevated renal pressures during routine flexible ureteroscopic stone manipulation. J Endourol 2004; 18:33-36.

5. Monga M. Bhayani S, Landman J, et al. Ureteral access for upper urinary tract disease: the access sheath. J. Endourol. 2001; 15, 831-834.

6. Huang J, Zhao Z, AlSmadi JK, et al. Use of the ureteral access sheath during ureteroscopy: A systematic review and metaanalysis. PLoS ONE 2018; 13(2): 0193600

7. Yuk HD, Park J, Cho SY. The effect of preoperative ureteral stenting in retrograde intrarenal surgery: multicenter, propensity score-matched study. BMC Urology 2020; 20:147-153.

8. L'Esperance JO, Ekeruo WO, Scales CD Jr, et al. Effect of ureteral access sheath on stone-free rates in patients undergoing ureteroscopic management of renal calculi. Urology 2005; 66:252-255.

9. Berquet G, Prunel P, Verhoest G, et al. The use of a ureteral access sheath does not improve stone-free rate after ureteroscopy for upper urinary tract stones. World J Urol 2014; 32:229-232.

10. 10- Forzini T, Lecuelle D, Alezra E, et al. Predictive factors of insertion failure of ureteral access sheath for flexible ureteroscopy: A study about 594 procedures. Eur Urol Suppl 2017; 16(3); e385.

11. Mogilevkin Y, Sofer M, Margel D, et al. Predicting an Effective Ureteral Access Sheath Insertion: A Bicenter Prospective Study. J Endourol 2014; 24(12): 14141417.

12. Alkhamees M, Aljuhayman A, Abdulmalik AA, et al. Failure of ureteral access sheath insertion in virgin ureters: A retrospective tertiary care center study. Urology Annals 2020; 12(4):331-334.

13. Traxer O, Thomas A. Prospective evaluation and classification of ureteral wall injuries resulting from insertion of a ureteral access sheath during retrograde intrarenal surgery. J Urol 2013; 189(2):580-4.

14. Astroza G, Catalan M, Consigliere L, et al. Is a ureteral stent required after use of ureteral access sheath in presented patients who undergo flexible ureteroscopy? Cent European J Urol 2017; 70:88-92.

15. Breda A, Emiliani E, Millan F, et al. The new concept of ureteral access sheath with guidewire disengagement: one wire does it all. World J Urol 2016; 34:603-6. 
16. Fuller TW, Rycyna KJ, Ayyash OM, et al. Defining the rate of primary ureteroscopic failure in unstented patients: A multi institutional study. J Endourol 2016; 30:970 4.

17. Koo KC, Yoon JH, Park NC, et al. The impact of preoperative $\alpha$-adrenergic antagonists on ureteral access sheath insertion force and the upper limit of force required to avoid ureteral mucosal injury: a randomized controlled study. J Urol 2018; 199:1622-30.

18. Traxer O, Wendt-Nordahl G, Sodha H, et al. Differences in renal stone treatment and outcomes for patients treated either with or without the support of a ureteral access sheath: the Clinical Research Office of the Endourological Society Ureteroscopy Global Study. World J Urol 2015; 33:2137-44.

19. Chew BH, Zavaglia B, Paterson RF, et al. A multicenter comparison of the safety and effectiveness of ureteroscopic laser lithotripsy in obese and normal weight patients. J Endourol 2013; 27:710-4.

20. Lildal SK, Sorensen FB, Andreassen KH, et al. Histopathological correlations to ureteral lesions visualized during ureteroscopy. World J Urol 2017; 35:1489-96.

21. Dinlenc CZ, Liatsikos EN, Smith AD. Ureteral ischemia model: an explanation of ureteral dysfunction after chronic obstruction. J Endourol 2002; 16:47-50.

22. Zelenko N, Coll D, Rosenfeld AT, et al. Normal ureter size on unenhanced helical CT. AJR Am J Roentgenol 2004; 182:1039-41.

23. Lildal SK, Nørregaard R, Andreassen KH, et al. Ureteral access sheath influence on the ureteral wall evaluated by cyclooxygenase- 2 and tumor necrosis factor- $\alpha$ in a porcine model. J Endourol 2017; 31:307- 13.

24. Patel N, Monga M. Ureteral access sheaths: a comprehensive comparison of physical and mechanical properties. Int Braz J Urol. 2018; 44(3):524-535. 
Figures and Tables

Figure 1. Shape of the UO over the stiff guidewire.

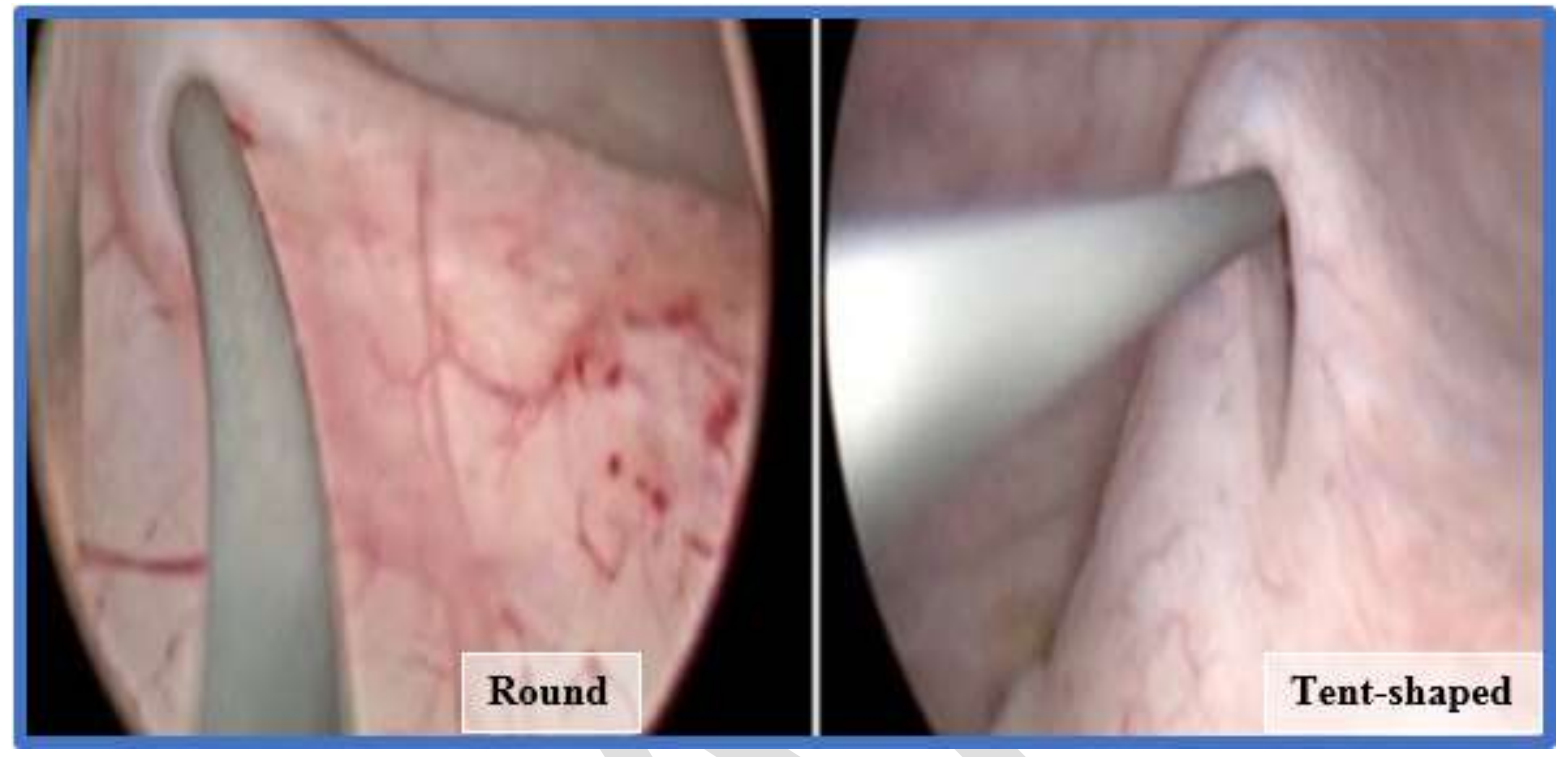




\begin{tabular}{|c|c|c|}
\hline \multicolumn{3}{|c|}{ Table 1. Overall characteristics of the study population } \\
\hline \multicolumn{2}{|c|}{ Variable $(n=128)$} & Mean (SD)/n (\%) \\
\hline \multicolumn{2}{|l|}{ Age/years } & $43.3 \pm 2.3$ \\
\hline \multicolumn{2}{|l|}{ Sex (males) } & $95(74.2 \%)$ \\
\hline \multicolumn{2}{|l|}{$\mathrm{BMI}\left(\mathrm{kg} / \mathrm{m}^{2}\right)$} & $30.0 \pm 6.9$ \\
\hline \multirow{4}{*}{ Comorbidities } & Diabetes & $26(20.3)$ \\
\hline & HTN & $23(18.0)$ \\
\hline & CVD & $10(7.8)$ \\
\hline & Others & $31(24.2)$ \\
\hline \multicolumn{2}{|l|}{ Stone burden $(\mathrm{mm})$} & $12.3 \pm 5.4$ \\
\hline \multicolumn{2}{|c|}{ Laterality (left-side) } & $80(62.5)$ \\
\hline \multicolumn{2}{|c|}{ Current use of alpha-blockers } & $49(38.3)$ \\
\hline \multicolumn{2}{|c|}{ History of spontaneous passage of stones } & $16(12.5)$ \\
\hline \multirow[t]{2}{*}{ Stone multiplicity } & Single kidney stones & $57(44.5)$ \\
\hline & Multiple kidney stones & $71(55.5)$ \\
\hline \multirow{2}{*}{$\begin{array}{l}\text { Shape of the UO } \\
(n=92)\end{array}$} & Round & $56(60.9)$ \\
\hline & Tent-shaped & $36(39.1)$ \\
\hline
\end{tabular}

BMI: body mass index; CVD: cardiovascular disease; HTN: hypertension; SD: standard deviation; UO: ureteral orifice.

\begin{tabular}{|c|c|c|c|c|}
\hline \multicolumn{5}{|c|}{$\begin{array}{l}\text { Table 2. Successful primary insertion of the UAS into virgin ureters or after } \\
\text { ureteral dilatation vs. a failed or aborted procedure }\end{array}$} \\
\hline \multicolumn{2}{|l|}{ Parameter } & $\begin{array}{l}\text { Successful } \\
(n=110)\end{array}$ & $\begin{array}{l}\text { Failure } \\
(n=18)\end{array}$ & $\mathbf{p}$ \\
\hline \multicolumn{2}{|c|}{ Mean age/years } & $48.3 \pm 8.2$ & $41.1 \pm 6.3$ & $<0.001$ \\
\hline \multicolumn{2}{|c|}{ Male sex } & $76(69.1)$ & $14(77.8)$ & 0.58 \\
\hline \multicolumn{2}{|c|}{ Mean BMI $\left(\mathrm{kg} / \mathrm{m}^{2}\right)$} & $27.2 \pm 4.6$ & $33.6 \pm 6.4$ & $<0.001$ \\
\hline \multicolumn{2}{|c|}{ Mean stone burden (mm) } & $12.3 \pm 3.2$ & $10.8 \pm 1.8$ & 0.06 \\
\hline \multicolumn{2}{|c|}{ Laterality (left-side) } & $57(51.8)$ & $14(77.8)$ & 0.04 \\
\hline \multicolumn{2}{|c|}{ Stone passage } & $14(12.7)$ & $2(11.1)$ & 1.00 \\
\hline \multicolumn{2}{|c|}{ Use of alpha-blockers } & $43(39.1)$ & $7(38.9)$ & 0.98 \\
\hline \multirow{2}{*}{$\begin{array}{l}\text { Stone } \\
\text { multiplicity }\end{array}$} & Single & $52(47.3)$ & $7(38.9)$ & \multirow[b]{2}{*}{0.61} \\
\hline & Multiple & $58(52.7)$ & $11(61.1)$ & \\
\hline \multirow{2}{*}{$\begin{array}{l}\text { Shape of } \\
\text { the UO over } \\
\text { the } \\
\text { guidewire } \\
(n=92)\end{array}$} & $\begin{array}{l}\text { Round } \\
(\mathrm{n}=56)\end{array}$ & $40(71.4)$ & $16(28.6)$ & \multirow[t]{2}{*}{0.007} \\
\hline & $\begin{array}{l}\text { Tent- } \\
\text { shaped } \\
(n=36)\end{array}$ & $34(94.4)$ & $2(5.6)$ & \\
\hline \multicolumn{2}{|l|}{ UO dilatation } & $29(26.4)$ & $6(33.3)$ & 0.57 \\
\hline
\end{tabular}

Results are expressed as means \pm standard deviations or as numbers $(\%)$. BMI: body mass index; UAS: ureteral access sheath; UO: ureteral orifice 


\begin{tabular}{|c|c|c|c|c|}
\hline \multicolumn{2}{|l|}{ Parameter } & $\begin{array}{l}\text { Success in } \\
\text { virgin } \\
\text { ureters } \\
(\mathrm{n}=81)\end{array}$ & $\begin{array}{l}\text { Success } \\
\text { after UC } \\
\text { dilation } \\
(\mathbf{n}=29) \\
\end{array}$ & $\mathbf{p}$ \\
\hline \multicolumn{2}{|c|}{ Mean age/years } & $47.4 \pm 6.5$ & $43.2 \pm 7.6$ & 0.005 \\
\hline \multicolumn{2}{|c|}{ Male sex } & $56(69.1)$ & $24(82.5)$ & 0.22 \\
\hline \multicolumn{2}{|c|}{ Mean BMI $\left(\mathrm{kg} / \mathrm{m}^{2}\right)$} & $28.8 \pm 5.9$ & $32.6 \pm 4.2$ & 0.002 \\
\hline \multicolumn{2}{|c|}{ Mean stone burden $(\mathrm{mm})$} & $11.8 \pm 4.1$ & $10.5 \pm 1.6$ & 0.10 \\
\hline \multicolumn{2}{|c|}{ Laterality (left-side) } & $42(51.9)$ & $15(51.7)$ & 1.00 \\
\hline \multicolumn{2}{|c|}{ Stone passage } & $10(12.3)$ & $4(13.8)$ & 0.98 \\
\hline \multicolumn{2}{|c|}{ Use of alpha-blockers } & $32(39.5)$ & $11(37.9)$ & 0.99 \\
\hline \multirow{2}{*}{$\begin{array}{l}\text { Stone } \\
\text { multiplicity }\end{array}$} & Single & $38(46.9)$ & $14(48.3)$ & \multirow[b]{2}{*}{1.00} \\
\hline & Multiple & $43(53.1)$ & $15(51.7)$ & \\
\hline \multirow{3}{*}{$\begin{array}{l}\text { Shape of } \\
\text { the UO over } \\
\text { the } \\
\text { guidewire } \\
(\mathrm{n}=74) \\
\text { Stone-free }\end{array}$} & $\begin{array}{l}\text { Round } \\
(\mathrm{n}=40)\end{array}$ & $20(35.7)$ & $20(69.0)$ & \multirow[t]{2}{*}{$<0.001$} \\
\hline & $\begin{array}{l}\text { Tent- } \\
\text { shaped } \\
(\mathrm{n}=34)\end{array}$ & $32(88.9)$ & $2(6.9)$ & \\
\hline & & $64(79.0)$ & $22(75.8)$ & 0.79 \\
\hline
\end{tabular}

Results are expressed as means \pm standard deviations or as numbers (\%). BMI: body mass index; UAS: ureteral access sheath; UO: ureteral orifice. 


\begin{tabular}{|l|l|l|}
\hline $\begin{array}{l}\text { Table 4. Multivariate regression analysis of predictors of UAS insertion into } \\
\text { virgin ureters before flexible ureteroscopy }\end{array}$ \\
\hline & OR $(\mathbf{9 5 \%}$ CI) & p \\
\hline Parameter & $1.01(0.84-1.04)$ & 0.589 \\
\hline Age of patients $>50$ years & $1.45(0.92-1.63)$ & 0.067 \\
\hline Male sex & $1.89(1.28-7.03)$ & 0.043 \\
\hline BMI <30 kg/m ${ }^{2}$ & $1.26(0.98-1.56)$ & 0.081 \\
\hline Spontaneous stone passage & $6.60(3.8-7.2)$ & 0.004 \\
\hline Tent-shaped UO over the guidewire & OR:
\end{tabular}

BMI: body mass; CI: confidence interval; OR: odds ratio; UO: ureteral orifice.

\begin{tabular}{|c|c|c|c|}
\hline Injury & & Endoscopic findings & n (\%) \\
\hline \multirow[b]{2}{*}{$\begin{array}{l}\text { Low- } \\
\text { grade }\end{array}$} & G0 & No lesion found or only mucosal petechiae & $101(91.8)$ \\
\hline & G1 & $\begin{array}{l}\text { Ureteral mucosal erosion without smooth } \\
\text { muscle injury }\end{array}$ & $7(6.4)$ \\
\hline \multirow[t]{3}{*}{$\begin{array}{l}\text { High- } \\
\text { grade }\end{array}$} & G2 & $\begin{array}{l}\text { Ureteral wall injury, including mucosa and } \\
\text { smooth muscle, with adventitial preservation } \\
\text { (periureteral fat not seen) }\end{array}$ & $2(1.8)$ \\
\hline & G3 & $\begin{array}{l}\text { Ureteral wall injury, including mucosa and } \\
\text { smooth muscle, with adventitial perforation } \\
\text { (periureteral fat seen) }\end{array}$ & $0(0 \%)$ \\
\hline & G4 & Total ureteral avulsion & $0(0 \%)$ \\
\hline
\end{tabular}

UAS: ureteral access sheath. 